\title{
Author Correction: Musashi 1 regulates the timing and extent of meiotic mRNA translational activation by promoting the use of specific CPEs
}

\author{
Laure Weill, Eulàlia Belloc, Chiara Lara Castellazzi and Raúl Méndez
}

Correction to: Nature Structural \& Molecular Biology https://www.nature.com/articles/nsmb.3434, published online 17 July 2017.

In the version of this article originally published, the legend to Supplementary Fig. 3 was incorrect (included text describing a panel not presented there (cited as b) and cited the wrong panel letter (c) for the text describing panel b). The correct legend is as follows: "Immature oocytes were injected with the indicated RNA constructs, then overexpressed HA-Msil or endogenous CPEB1 were immnoprecipitated. The IPs were analyzed by western blot and by RT qPCR. Anti-HA IP without HA-Msil overexpression and IgG IP were used as controls. (a) Left panel, IP HA-Msil analyzed by western blot. Right panel, qPCRs of the indicated reporters performed using reversed transcribed immunoprecipitated mRNAs. Input was normalized against endogenous GAPDH mRNA, and IP efficiency, against endogenous c-mos mRNA. (b) Translational regulation of Mos $(+12)$ and non-consensus CPE containing reporters. Synthetic mRNAs containing the firefly luciferase coding sequence fused to the indicated 3' UTRs were co-injected with control renilla luciferase mRNA. Oocytes were collected $2 \mathrm{~h}$ after GVBD to determine luciferase activity. Schematic representations of each $3^{\prime}$ UTR are shown to the left, with consensus CPEs (C) depicted as blue boxes; non-consensus CPEs, green boxes; MBEs (M), orange circles; PAS (H), red hexagons; and mutated CPE, MBE or PAS, as an empty box, circle or hexagon, respectively; distance between elements is given in nucleotides."

Also, the directions (left, right) in the legend for Supplementary Fig. 4a were incorrect; the correct panel legend is as follows: “(a) Effects of MBEb mutation on CPEB1 binding. Immature oocytes were injected with the indicated constructs. Upper panel, IP against CPEB1 analyzed by western blot; lower panel, qPCR of the indicated reporter after reverse transcription of the immunoprecipitated mRNAs (data, mean \pm SEM; $n=3)$."

Published online: 12 July 2019

https://doi.org/10.1038/s41594-019-0272-4 